\title{
BMJ Open Acupuncture for breathlessness in advanced cancer: a protocol for systematic review and meta-analysis with trial sequential analysis
}

\author{
Zihan Yin (D) , Tao Xu, Mingsheng Sun (D) , Ling Zhao, Fanrong Liang (d)
}

To cite: Yin Z, Xu T, Sun M, et al. Acupuncture for breathlessness in advanced cancer: a protocol for systematic review and meta-analysis with trial sequential analysis. BMJ Open 2021;11:e054917. doi:10.1136/ bmjopen-2021-054917

- Prepublication history for this paper is available online. To view these files, please visit the journal online (http://dx.doi. org/10.1136/bmjopen-2021054917).

Received 26 June 2021 Accepted 26 October 2021

Check for updates

(C) Author(s) (or their employer(s)) 2021. Re-use permitted under CC BY-NC. No commercial re-use. See rights and permissions. Published by BMJ.

School of Acu-Mox and Tuina, Chengdu University of Traditional Chinese Medicine, Chengdu, Sichuan, China

Correspondence to Fanrong Liang; acuresearch@126.com and Professor Ling Zhao; zhaoling@cdutcm.edu.cn

\section{ABSTRACT}

Introduction Breathlessness in advanced cancer, a frequent multicomponent and debilitating disorder, severely reduces function and quality of patients' life. Multiple studies have shown that non-pharmacological therapies can effectively palliate breathlessness in advanced cancer. However, no systematic review has investigated the application of acupuncture, as a non-pharmacological treatment, for breathlessness in advanced cancer. A systematic review will be conducted to summarise evidence supporting the efficacy and safety of acupuncture as a therapeutic option for breathlessness in advanced cancer based on existing randomised controlled trials (RCTs).

Methods RCTs will be retrieved from nine scientific databases, including the MEDLINE via PubMed, Web of Science via the Web of Knowledge, Embase via Ovid, the Cochrane Central Register of Controlled Trials via the Cochrane Library, and Allied and Complementary Medicine Database via EBSCO, China National Knowledge Infrastructure, Wanfang Database, VIP Database, Chinese Biomedical Literature Database; three clinical registry platforms, including the WHO International Clinical Trials Registry Platform, NIH Clinical trials.gov and Chinese Clinical Trial Registry, as well as from other sources. Studies published since inception of these databases to 1 August 2021 will be retrieved. Search terms will include breathlessness, cancer, acupuncture and RCT. Two investigators will independently select and extract data from RCTs and assess the risk of bias. The primary outcome, which is alleviation of breathlessness, will be assessed. Metaanalysis will be performed using RevMan V.5.4 and STATA V.15.0. The TSA 0.9.5.10 $\beta$ software will be used to conduct trial sequential analysis. Finally, the quality of evidence from RCTs will be assessed using the Grading of Recommendations Assessment, Development and Evaluation System tool. Ethics and dissemination Results will be disseminated through peer-reviewed journals or conference reports. Since this study involves acquisition of secondary data, ethical approval requirements will be waived. PROSPERO registration number CRD42021240085.

\section{INTRODUCTION}

Description of the condition

Breathlessness, shortness of breath and dyspnoea are synonyms used to describe the 'subjective experience of breathing discomfort', 12 and are the most distressing symptoms
Strengths and limitations of this study

- This review will be the first meta-analysis to summarise the efficacy and safety of acupuncture for the treatment of breathlessness in advanced cancer.

- All study procedures will strictly follow the Preferred Reporting Items for Systematic Reviews and Metaanalyses guideline.

- Trial sequential analysis will be used to estimate the required information size for meta-analysis.

- The quality of evidence from randomised controlled trials will be evaluated using the Grading of Recommendations Assessment, Development and Evaluation Systems tool.

- Various acupuncture methods, acupoints and control treatments may cause considerable heterogeneity.

experienced by patients with advanced cancer. ${ }^{3}$ It has been reported that 10\%-70\% of patients diagnosed with advanced cancer experience significant breathlessness. ${ }^{4-7}$ As a multidimensional, frequent and debilitating symptom of advanced cancer, breathlessness has a negative impact on respiratory function. ${ }^{8} 9$ In addition to obvious functional limitations seen in patients with advanced cancer, it negatively affects the long-term quality of patient's life and mind and is a significant prognostic factor of advanced cancer. $^{10}$

\section{Description of the intervention}

While both pharmacological $^{11}$ and nonpharmacological treatments ${ }^{8}$ may control breathlessness in patients with advanced cancer, there is no gold-standard therapy for managing breathlessness in advanced cancer due to various factors (cancer type, cancer phase, pathophysiologic mechanism, etc).$^{12} 13$ As a non-pharmacological therapy, ${ }^{8}$ acupuncture has been shown to improve breathlessness in patients with advanced cancer in China. Randomised controlled trials (RCTs) ${ }^{14-16}$ have shown that acupuncture 
can alleviate breathlessness in advanced cancer. For instance, Minchom et $a l^{14}$ found that acupuncture effectively controlled breathlessness, anxiety and morphine consumption in patients with advanced cancer.

\section{How the intervention might work}

The underlying mechanisms of acupuncture on breathlessness have been covered in previous studies. ${ }^{17-19}$ Acupuncture may target various aspects of the pathological process of breathlessness such as central role of endogenous opioid peptide release,${ }^{20}$ anti-inflammatory effect of epinephrine corticosteroid release ${ }^{21}$ and psychological effects. ${ }^{22}$

\section{Why it is important to perform this review}

Nowadays, there is no approved therapy to control breathlessness in advanced cancer. Acupuncture has been applied for many years to treat breathlessness in advanced cancer in China. A previous review ${ }^{8}$ evaluated the efficacy of non-pharmacological methods in the treatment of breathlessness in advanced cancer, but it did not comprehensively describe the benefits of acupuncture therapy. Moreover, no systematic review/meta-analysis (SRs/MAs) has been reported regarding the safety and efficacy of acupuncture in treating breathlessness in advanced cancer. Therefore, we designed this SR/MA to provide evidence on the safety and efficacy of acupuncture therapy in the management of breathlessness in advanced cancer.

\section{OBJECTIVES}

To evaluate the efficacy and safety of acupuncture in the treatment of breathlessness in patients with advanced cancer and inform clinical and health policy decisions.

\section{METHODS}

The protocol is written according to the Preferred Reporting Items for Systematic Reviews and MetaAnalyses Protocol (PRISMA-P) guidelines. ${ }^{23}$ The review will also follow the PRISMA guidelines ${ }^{24}$ and A Measurement Tool to Assess systematic Reviews-2 (AMSTAR-2). ${ }^{25}$ The study will start on 1 August 2021, and will end on 1 December 2021.

\section{Eligibility criteria}

Types of studies

The study will include original published full-text RCTs written in Chinese/English without any region restrictions.

\section{Types of participants}

This study will enrol patients diagnosed as advanced cancer with breathlessness. Participants with all types of advanced cancer diagnosed using any recognised criteria will be included regardless of age, gender, nationality, ethnicity and aetiology.
Types of interventions

Acupuncture approaches such as manual acupuncture, electronic acupuncture, acupuncture-moxibustion, warm acupuncture, fire acupuncture, ear acupuncture and transcutaneous electrical acupoint stimulation, as monotherapies or additional methods, regardless of stimulation approaches, acupoint selection and additional therapies, will be included. ${ }^{26}$

\section{Types of control group}

The control group will include conventional medicine treatment, placebo, usual care, waiting-list and other similar interventions.

Types of outcome measures

Primary outcomes

The acceptable subjective outcome will be alleviation of breathlessness after acupuncture treatment. Quantitative unidimensional validated tools, such as Visual Analogue Scale, Numerical Rating Scale, Borg Scale, will be used to assess the severity of breathlessness.

\section{Secondary outcomes}

1. Exercise tolerance, which will be assessed through the 6-min walk test.

2. Quality of life, which will be assessed through the European Organisation for Research and Treatment of Cancer Quality of Life Questionnaire (EORTC QLQ), the general version of Functional Assessment of Cancer Therapy (FACT), KPS (Karnofsky Performance Status) and other validated questionnaires.

3. Psychological improvements, which will be assessed through the Hamilton Anxiety Scale-24, Self-rating Anxiety Scale, Hamilton Depression Scale-24, Selfrating Depression Scale, etc.

4. Safety, assessed by directly describing adverse events associated with intervention approaches.

\section{Exclusion criteria}

The studies meeting any one of following exclusion criteria will be excluded.

1. Non-RCTs, qualitative studies, case reports, conference abstracts, expert experience, letters, comments, animal studies, duplicated articles will be excluded.

2. Incomplete research information.

3. Studies not published in Chinese/English.

\section{Search strategy}

Studies published since inception to 1 August 2021 will be retrieved from the following databases: Chinese Biomedical Literature Database (CBM), China National Knowledge Infrastructure (CNKI), Wanfang Database (WF), VIP Database, Web of Science (WOS) (1965-2021) via the Web of Knowledge, Embase (1974-2021) via Ovid, MEDLINE (1966-2021) via PubMed, the Cochrane Central Register of Controlled Trials (CENTRAL) (The Cochrane Library, 2021, Issue 8) and Allied and Complementary Medicine Database (AMED) (1985-2021) via EBSCO. Data from clinical registries (The World Health 


\section{Box 1 Search strategy for the PubMed database}

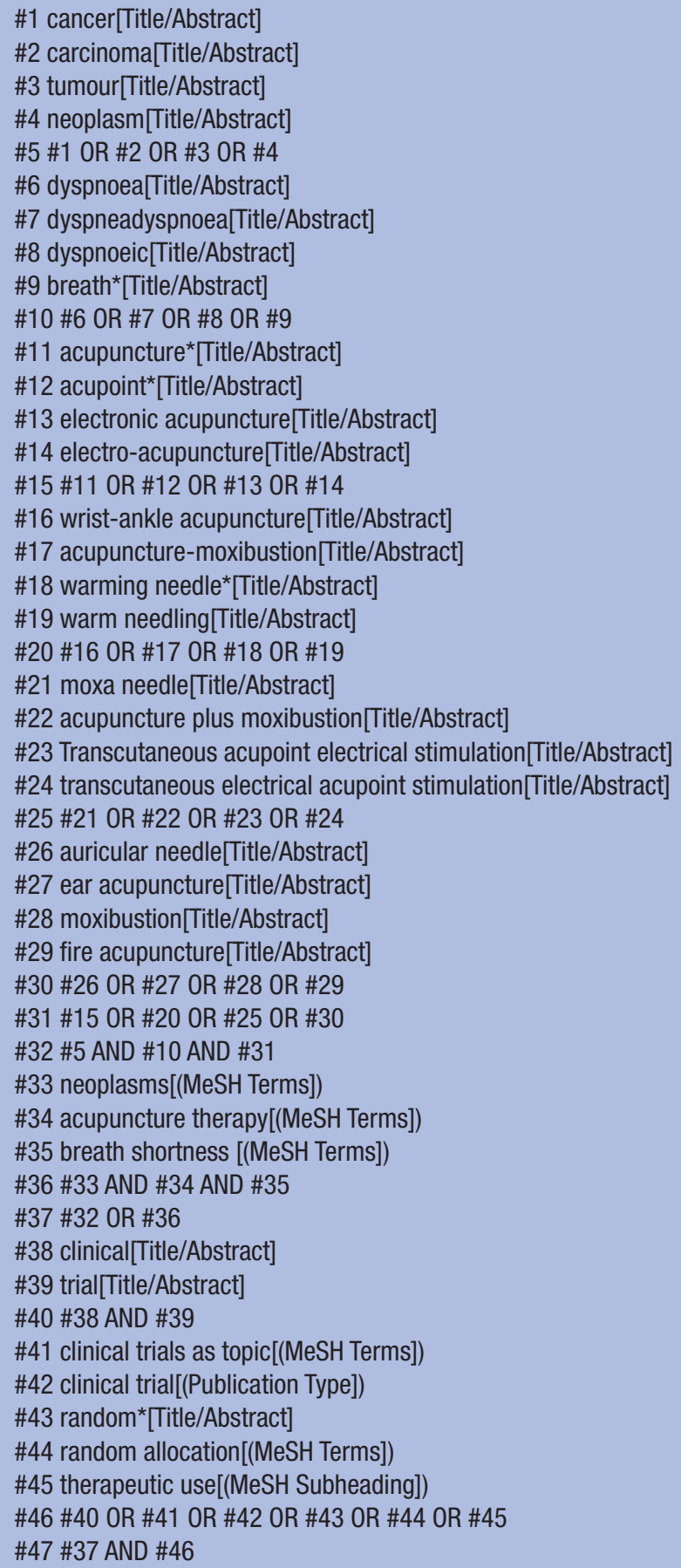

Organisation International Clinical Trials Registry Platform (WHO ICTRP), National Institutes of Health clinical registry (Clinical trials.gov) and Chinese Clinical Trial Register (ChiCTR)) will also be retrieved to minimise the risk of publication bias. Moreover, data will be obtained from other similar published SRs/MAs.

The PubMed search strategy is shown in box 1. Search terms to be used will be: cancer, neoplasm, breathlessness, dyspnoea, acupuncture, acupoint, random, trial, etc. These terms will be used alone or in combinations.
These words will be connected using 'and', 'or'. Chinese retrieval modes will be similar to English retrieval.

\section{Study selection and data extraction}

Two researchers (ZY and TX) will be trained to independently extract data. Intraclass correlation coefficient will be used to test the consistency of data obtained by the two researchers.

During study selection, they will first exclude duplicate studies using the NoteExpress V.3.0. They will then assess the titles/abstracts of identified studies to exclude unmatched studies according to the inclusion criteria. Finally, the two researchers will each read the full-text of the remaining studies to select those that meet the inclusion criteria. Any divergences in the data obtained by the two researchers will be solved through discussion between them, if a clear answer is not obtained, a third party (LZ or FL) will help to reach a final decision.

Data extraction will be accomplished by the same reviewers using a standardised data extraction form. Disagreements will be resolved by a third party (LZ or FL). In case of missing or incomplete information in any of the RCTs, the original corresponding/first author of the study will be contacted. If such data are difficult to be acquired, it will be declared in our study. The researchers will enter the information into a data extraction form. The form shall contain the following general information: name of the first author, publication year and source, country/region, study design, age, gender, diagnostic criteria, sample size, intervention group details, control group details, outcomes and results. Details of acupuncture therapy will be covered according to Standards for Reporting Interventions in Clinical Trials of Acupuncture (STRICTA) ${ }^{27}$ The entire study selection process for this review is shown in figure 1 .

\section{Quality assessment}

The Cochrane Collaborative Bias Risk Tool $^{28}$ will be used to evaluate the methodological quality of the included RCTs. The two researchers (ZYand TX) will independently evaluate the quality of these studies using the Cochrane Handbook for Systematic Reviews of Interventions, which contains seven elements (sequence generation, allocation concealment, participant blinding, blindness of result evaluators, incomplete outcome data, selective outcome reporting and other biases). Intraclass correlation coefficient will also be calculated to test consistency in the data obtained by the two researchers. If necessary, corresponding authors (LZ and FL) will help us judge the consistency. The risk of bias will be classified as low risk, unclear risk or high risk. Review Manager (RevMan) V.5.4 will be used to generate the figure of risk of bias.

\section{Measures of treatment effect}

STATA V.15.0 will be used for data analysis. For the included RCTs, we will implement prepost differences or end-point scores as outcomes. For dichotomous data, risk ratios with $95 \%$ CIs will be calculated. Weighted mean 


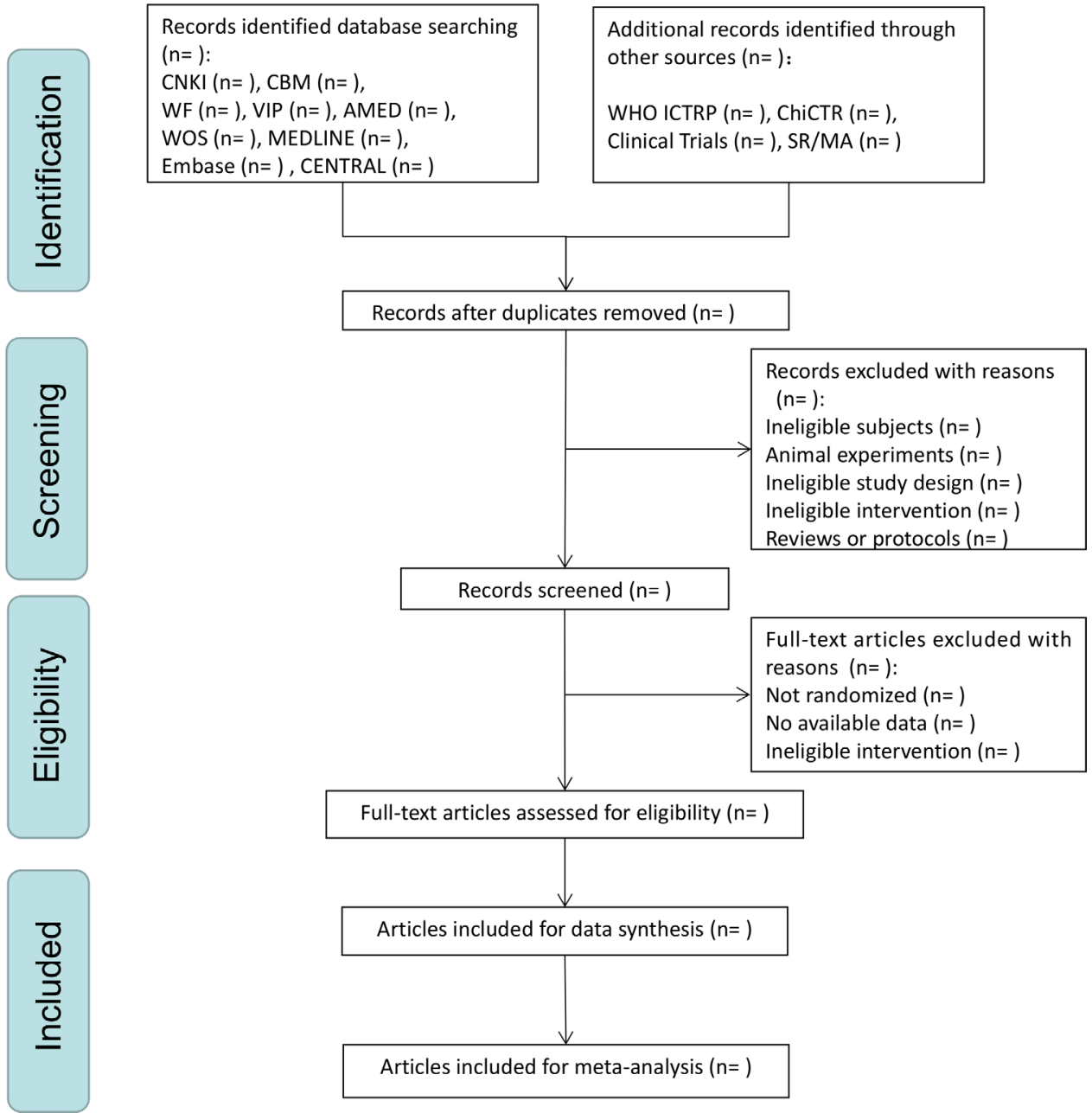

Figure 1 The selection process flow chart. AMED, Allied and Complementary Medicine Database; CBM, Chinese Biomedical Literature Database; CENTRAL, the Cochrane Central Register of Controlled Trials; ChiCTR, Chinese Clinical Trial Register; CNKI, China National Knowledge Infrastructure; SR/MA, systematic review/meta-analysis; WF, Wanfang Database; WHO ICTRP, World Health Organisation International Clinical Trials Registry Platform; WOS, Web of Science.

differences or standardised mean differences with 95\% CI will be calculated for continuous data. Fixed-effects model will be conducted based on the Mantel-Haenszel method. Otherwise, the random-effects model will be carried out using the Der Simonian-Laired method. $\mathrm{P} \leq 0.05$ will be set as the threshold for statistical significance.

\section{Dealing with missing data}

Attempts will be made to contact the corresponding authors to acquire missing data, where feasible. If the missing information are unattainable, the worst-case scenario analysis, extreme worst-case analysis, extreme best-case analysis ${ }^{29}$ will be carried out to assess the latent impact.

\section{Assessment of heterogeneity}

Testing clinical/methodological heterogeneity will concentrate on patients' details, intervention type, outcomes of included RCTs and comparison of goodness of fit between fixed-effects and random-effects model. $I^{2} / X^{2}$ will be selected to identify statistical heterogeneity across studies in all analyses based on Cochrane Handbook V.5.4.0. The fixed-effect model will be used for analysis when $\mathrm{p} \geq 0.1$, and $I^{2} \leq 50 \%$. Otherwise, the randomeffects model will be used.

\section{Subgroup analysis}

In case of potential heterogeneity, subgroup analysis will be rationally performed according to the type of cancer, acupuncture methods, acupoints, control treatments and outcomes among others.

\section{Sensitivity analysis}

Sensitivity analyses will be performed to determine the robustness and quality of results. According to the Cochrane handbook, methodological quality, sample size and data analysis techniques will be used to assess sensitivity. Where heterogeneity source will not be established, narrative description will be done to illustrate it, in which certain assumptions will be made regarding accuracy and depth of the results.

\section{Other analysis}

In case substantial heterogeneity is found, we will use a meta-regression approach to evaluate the potential heterogeneity. 


\section{Assessment of publication bias}

If necessary, funnel plot analysis will be used for digitalbased modelling of the results. Potential publication biases will be explored using Egger's test.

\section{Trial sequential analysis}

Trial sequential analysis $(\mathrm{TSA})^{30}$ applies the design principle of group sequential, which helps to adjust the random error of meta-analysis, and estimate the required information size (RIS) for meta-analysis to reduce the risk associated with repeated statistical tests. TSA will be performed using the TSA 0.9.5.10 $\beta$ software (Copenhagen Trial Unit, Copenhagen, Denmark). Statistical indices for the RIS estimate will be 0.05 for type I error $(\alpha)$ and 0.2 for type II errors $(\beta)$.

\section{Quality of evidence}

The Grades of Recommendations, Assessment, Development and Evaluation (GRADE) tool ${ }^{31}$ will be used to evaluate evidence quality. Two investigators, who will have been trained on GRADE, will independently perform quality assessment of the evidence. Based on GRADE, quality level will be classified as high, moderate, low or critically low.

\section{Patient and public involvement}

No participant will be directly involved in this study. Only data in RCTs from the above sources will be used for the analyses.

\section{DISCUSSION}

Numerous studies have documented the efficacy and safety of acupuncture in the treatment of breathlessness in patients with advanced cancer. However, no evidence has been provided to show the efficacy and safety of acupuncture in the management of breathlessness in advanced cancer. To address these limitations, we have designed an SR/MA to summarise the existing evidence on the efficacy and safety of acupuncture in alleviating breathlessness in advanced cancer. Findings from this study will inform future studies and clinical decisions.

\section{ETHICS AND DISSEMINATION}

The results will be disseminated via peer-reviewed publication. Since no private and confidential patient data will be included, the SR does not require ethical clearance.

Acknowledgements The authors thank the National key research and development program of China, National Natural Science Foundation of China and the Project of science and Technology Department of Sichuan Province for financial support. We also thank Home for Researchers (www.home-for-researchers.com) for editing the English text of a draft of this manuscript.

Contributors ZY, FL and LZ conceived the study. ZY and MS will develop the study protocol and implement the systematic review under the supervision of $L Z$ and FL. ZY and TX will design statistical analyses and carry out statistical analyses. $Z Y$ and $T X$ will perform literature search, screening, data extraction and risk of bias assessment. FL and LZ will supervise the work. ZY wrote the first draft of the manuscript. All authors contributed to the drafting of the final protocol.
Funding This work was financially supported by the National key research and development program of China (no. 2019YFC1709700), the National Natural Science Foundation of China (no. 81590951, 82004486, 81722050, 81973961), and the Project of science and Technology Department of Sichuan Province (20ZDYF1199, 2019YFS0081).

Competing interests None declared.

Patient and public involvement Patients and/or the public were not involved in the design, or conduct, or reporting or dissemination plans of this research.

Patient consent for publication Not required.

Provenance and peer review Not commissioned; externally peer reviewed.

Open access This is an open access article distributed in accordance with the Creative Commons Attribution Non Commercial (CC BY-NC 4.0) license, which permits others to distribute, remix, adapt, build upon this work non-commercially, and license their derivative works on different terms, provided the original work is properly cited, appropriate credit is given, any changes made indicated, and the use is non-commercial. See: http://creativecommons.org/licenses/by-nc/4.0/.

\section{ORCID iDs}

Zihan Yin http://orcid.org/0000-0002-1543-0551

Mingsheng Sun http://orcid.org/0000-0002-6748-5087

Fanrong Liang http://orcid.org/0000-0001-8518-9268

\section{REFERENCES}

1 Parshall MB, Schwartzstein RM, Adams L, et al. An official American thoracic Society statement: update on the mechanisms, assessment, and management of dyspnea. Am J Respir Crit Care Med 2012;185:435-52.

2 Tishelman C, Petersson L-M, Degner LF, et al. Symptom prevalence, intensity, and distress in patients with inoperable lung cancer in relation to time of death. $J$ Clin Oncol 2007;25:5381-9.

3 Henson LA, Maddocks M, Evans C, et al. Palliative care and the management of common distressing symptoms in advanced cancer: pain, breathlessness, nausea and vomiting, and fatigue. J Clin Oncol 2020;38:905-14.

4 Hui D, Bohlke K, Bao T, et al. Management of dyspnea in advanced cancer: ASCO guideline. J Clin Oncol 2021;39:JCO2003465.

5 Solano JP, Gomes B, Higginson IJ. A comparison of symptom prevalence in far advanced cancer, AIDS, heart disease, chronic obstructive pulmonary disease and renal disease. J Pain Symptom Manage 2006;31:58-69.

6 Reuben DB, Mor V. Dyspnea in terminally ill cancer patients. Chest 1986;89:234-6.

7 Donnelly S, Walsh D. The symptoms of advanced cancer. Semin Oncol 1995;22:67-72.

8 Gupta A, Sedhom R, Sharma R, et al. Nonpharmacological interventions for managing breathlessness in patients with advanced cancer: a systematic review. JAMA Oncol 2021;7:290-8.

9 Hui D, Maddocks M, Johnson MJ, et al. Management of breathlessness in patients with cancer: ESMO clinical practice guidelines. ESMO Open 2020;5:e001038.

10 Cuervo Pinna MA, Mota Vargas R, Redondo Moralo MJ, et al. Dyspnea--a bad prognosis symptom at the end of life. Am J Hosp Palliat Care 2009;26:89-97.

11 Feliciano JL, Waldfogel JM, Sharma R, et al. Pharmacologic interventions for breathlessness in patients with advanced cancer: a systematic review and meta-analysis. JAMA Netw Open 2021;4:e2037632.

12 Levy M, Smith T, Alvarez-Perez A, et al. Palliative care version 1.2016. J Nat/ Compr Canc Netw 2016;14:82-113.

13 SM D, Gupta A, Waldfogel JM. Interventions for breathlessness in patients with advanced cancer. Rockville, MD: Agency for Healthcare Research and Quality (US), 2020.

14 Minchom A, Punwani R, Filshie J, et al. A randomised study comparing the effectiveness of acupuncture or morphine versus the combination for the relief of dyspnoea in patients with advanced non-small cell lung cancer and mesothelioma. Eur J Cancer 2016;61:102-10.

15 Filshie J, Penn K, Ashley S, et al. Acupuncture for the relief of cancerrelated breathlessness. Palliat Med 1996;10:145-50.

16 Vickers AJ, Feinstein MB, Deng GE, et al. Acupuncture for dyspnea in advanced cancer: a randomized, placebo-controlled pilot trial [ISRCTN89462491]. BMC Palliat Care 2005;4:5.

17 von Trott P, Oei SL, Ramsenthaler C. Acupuncture for Breathlessness in Advanced Diseases: A Systematic Review and Meta-analysis. J Pain Symptom Manage 2020;59:327-38. 
18 Fernández-Jané C, Vilaró J, Fei Y, et al. Acupuncture techniques for COPD: a systematic review. BMC Complement Med Ther 2020;20:138.

19 Bauml J, Haas A, Simone CB, et al. Acupuncture for dyspnea in lung cancer: results of a feasibility trial. Integr Cancer Ther 2016;15:326-32.

20 Filshie J, Rubens C. Acupuncture in palliative care. Acupunct Med 2011;29:166-7.

21 Kvorning N, Akeson J. Plasma adrenaline increases in anesthetized patients given electro-acupuncture before surgery. Pain Med 2010;11:1126-31.

22 Mukaino Y, Park J, White A, et al. The effectiveness of acupuncture for depression--a systematic review of randomised controlled trials. Acupunct Med 2005;23:70-6.

23 Shamseer L, Moher D, Clarke M, et al. Preferred reporting items for systematic review and meta-analysis protocols (PRISMA-P) 2015: elaboration and explanation. BMJ 2015;349:97647.

24 Moher D, Liberati A, Tetzlaff J, et al. Preferred reporting items for systematic reviews and meta-analyses: the PRISMA statement. BMJ 2009;339:b2535.

25 Pieper D, Puljak L, González-Lorenzo M, et al. Minor differences were found between AMSTAR 2 and ROBIS in the assessment of systematic reviews including both randomized and nonrandomized studies. J Clin Epidemiol 2019;108:26-33.

26 Yin Z, Geng G, Xu G, et al. Acupuncture methods for allergic rhinitis: a systematic review and Bayesian meta-analysis of randomized controlled trials. Chin Med 2020;15:109.

27 MacPherson H, Altman DG, Hammerschlag R, et al. Revised standards for reporting interventions in clinical trials of acupuncture (stricta): extending the CONSORT statement. Acupunct Med 2010;28:83-93.

28 Higgins JPT, Altman DG, Gøtzsche PC, et al. The Cochrane Collaboration's tool for assessing risk of bias in randomised trials. BMJ 2011;343:d5928.

29 Zhai J, Mu W, Si J, et al. Acupuncture for constipation in patients with stroke: protocol of a systematic review and meta-analysis. BMJ Open 2018;8:e020400.

30 Zhang Y-J, Cao H-J, Li X-L, et al. Cupping therapy versus acupuncture for pain-related conditions: a systematic review of randomized controlled trials and trial sequential analysis. Chin Med 2017;12:21

31 Guyatt GH, Oxman AD, Schünemann HJ, et al. GRADE guidelines: a new series of articles in the Journal of clinical epidemiology. J Clin Epidemiol 2011;64:380-2. 